\title{
Hämatoonkologische Neoplasien
}

\section{Die Lunge leidet mit}

\author{
Bei 40 bis $60 \%$ der Patienten mit malignen hämato- \\ logischen Erkrankungen treten bronchopulmonale \\ Veränderungen auf. Darunter fallen bronchopulmonale \\ Manifestationen der Grunderkrankung, Infektionen, \\ Medikamentennebenwirkungen und Zweit- \\ erkrankungen.
}

\section{Pulmonale Infiltration bei Morbus Hodgkin.}

- Nach einer eigenen retrospektiven Untersuchung von 250 Patienten, die Priv.-Doz. Dr. Jens Schreiber aus Magdeburg vorstellte, stehen unter den bronchopulmonalen Veränderungen bei hämatoonkologischen Patienten Infektionen mit 59\% im Vordergrund. An zweiter Stelle rangieren mit 19\% pneumotoxische Zytostatikareaktionen; 14\% machen die Manifestationen der Grunderkrankung (etwa zur Hälfte Erstmanifestationen und Rezidive) und 8\% Zweiterkrankungen aus.

\section{Primäre pulmonale Lymphome seltener als sekundäre}

Die Lunge ist ein ausgesprochen immunkompetentes Organ, das nicht nur Lymphknoten im Mediastinum und in den Hili aufweist, sondern auch mit einem eigenen lymphatischen System, dem BALT (bronchus-associated lymphoid tissue) ausgestattet ist. Primäre pulmonale Non-Hodgkin-Lymphome (NHL) kommen jedoch selten vor. Noch am häufigsten sind low-grade B-ZellLymphome (BALT-Lymphome). Sie verursachen oft keine Symptome und werden zufällig entdeckt. Einige Patienten fallen auch durch B-Symptomatik oder respiratorische Symptome auf. Meist handelt es sich um solitäre Rundherde. Sie können zu diffuser Infiltration des Parenchyms führen. Wird die Bronchialschleimhaut infiltriert, drohen Stenosierung, Atelektasen oder poststenotische Pneumonien.

Bis zu $20 \%$ der primären pulmonalen Lymphome sind high-grade NHL. Auch sie treten meist als Rundherde in Erscheinung. Sie führen zu teilweise ausgedehnten Schleimhautinfiltrationen und rufen in der Regel pulmonale und systemische Symptome hervor. Wesentlich häufiger als primäre NHL kommt ein pulmonaler Befall bei disseminierter Erkrankung vor, der immerhin jeden zweiten NHL-Patienten im Verlauf der Erkrankung betrifft.

Während mediastinale und hiläre Lymphknoten bei NHL selten betroffen sind, manifestiert sich der Morbus Hodgkin überwiegend mediastinal. Eine primäre pulmonale Lokalisation findet man jedoch extrem selten. Bei fortgeschrittener disseminierter Erkrankung ist die Lunge in rund $12 \%$ der Fälle in Form von solitären Rundherden, die einschmelzen können, miliaren Herden sowie Infiltration der Bronchialschleimhaut und des Interstitiums beteiligt.

Beim Plasmozytom sind Lunge und Pleura selten involviert, und wenn, dann kommt es meist spät im Krankheitsverlauf zu einer Infiltration mit Plasmazellen.

\section{Verstopfung der Lungenkapillaren}

Leukämiepatienten entwickeln in bis zu $80 \%$ der Fälle pulmonale Komplikationen. Meist handelt es sich um Infektionen und Medikamentennebenwirkungen. Im fortgeschrittenen Stadium können sich leukämische Infiltrate des Parenchyms und der Bronchialschleimhaut bilden, besonders bei hoher Blastenzahl im Blut. Die meist miliare Be- herdung bedingt eine respiratorische Insuffizienz.

Ein Leukostasesyndrom bei hoher Zellzahl kann auch die kapilläre pulmonale Strombahn verstopfen und damit den Gasaustausch massiv behindern. Die Patienten leiden unter Dyspnoe, es können sich Zyanose, Lungenödem, diffuser Alveolarschaden mit Hämorrhagie und eine pulmonale Hypertonie entwickeln. Meist tritt das Leukostasesyndrom bei der CML auf, weil die Zellen relativ groß sind. Die Therapie besteht in einer sofortigen Leukapherese. Auch chronische myeloproliferative Erkrankungen können direkt zu einer Verstopfung bzw. Thrombosierung der pulmonalen Gefäße führen.

Bildgebende Verfahren erlauben selten eine klare Zuordnung. Allerdings ist bei leukämischen oder Lymphominfiltraten im Unterschied zu soliden Tumoren die Bronchusanatomie meist erhalten. Besonders bei diffusen Prozessen bietet sich in vielen Fällen die bronchoalveoläre Lavage an. Sie erlaubt, die Klonalität der Zellpopulation nachzuweisen und mikrobiologische Befunde zu erheben. Wann immer möglich, sollte eine frühzeitige bronchoskopische Diagnostik mit Biopsie angestrebt werden, so Schreiber.

\section{Dr. med. Angelika Bischoff}

51. Kongress der DGP, Hannover 